\title{
Preliminary assessment of sandwich plates subject to blast loads
}

\author{
Zhenyu Xue, John W. Hutchinson* \\ Division of Engineering and Applied Sciences, Harvard University, Cambridge, MA 02138, USA
}

Received 8 July 2002; received in revised form 22 April 2003; accepted 9 May 2003

\begin{abstract}
The question motivating the present study is whether metal sandwich plates with sufficiently strong cores are able to sustain substantially larger blast loads than monolithic solid plates of the same material and total mass. Circular plates clamped at their edges are considered under blast loads large enough to produce substantial deflections. The material is elastic-perfectly plastic. Material strain-rate dependence and fracture are neglected. A dynamic finite element formulation for elastic-plastic solids is employed to analyze the plate response. Uniformly distributed blast impulses are considered. As a basis for comparison, complete results are obtained for solid plates for both zero-period and finite-period impulses. Similar computations are carried out for a set of sandwich plates having tetragonal truss cores. The potential for superior strength and energy absorbing capacity of the sandwich plates is demonstrated compared with solid plates having the same mass. The importance of both the strength and energy absorbing capacity of the core are highlighted for superior blast resistance. Proposals for further research are made.
\end{abstract}

(C) 2003 Elsevier Ltd. All rights reserved.

Keywords: Sandwich plates; Lightweight structure; Truss cores; Blast loading

\section{Introduction}

The superior performance of sandwich plates relative to monolithic solid plates in applications requiring high strength and stiffness is well known. Recent work on polymer matrix fiber-reinforced sandwich plates has focused on the advantages of this type of construction under blast loads when the deformations remain dominantly elastic [1]. The relative advantage of sandwich plates over solid plates has not been firmly established for metal construction when strong blast loads require both high strength and energy absorption. In this first paper of a series, the responses of metal sandwich plates

\footnotetext{
* Corresponding author.

E-mail address: webmaster@harvard.edu (J.W. Hutchinson).
} 


\begin{tabular}{|c|c|}
\hline \multicolumn{2}{|c|}{ Nomenclature } \\
\hline E & Young's modulus \\
\hline$E_{c}$ & equivalent Young's modulus of sandwich core \\
\hline$H_{c}$ & height (thickness) of sandwich core \\
\hline$h$ & thickness of solid plate \\
\hline$h_{f}$ & thickness of face sheet of sandwich plate \\
\hline$I$ & initial impulse \\
\hline$\hat{I}$ & impulse per unit area \\
\hline$K E_{\text {sandwich }}$ & initial kinetic energy imparted to sandwich plate \\
\hline$K E_{\text {solid }}$ & initial kinetic energy imparted to solid plate \\
\hline$L_{c}$ & length of truss member \\
\hline$M_{\text {sandwich }}$ & total mass of sandwich plate \\
\hline$M_{\text {solid }}$ & total mass of solid plate \\
\hline$p$ & pressure \\
\hline$R$ & radius of plate \\
\hline$R_{c}$ & radius of truss member \\
\hline$T$ & response duration of the plate \\
\hline$V_{0}$ & initial velocity \\
\hline$\alpha$ & material constant in metal foam \\
\hline$\delta_{\max }$ & maximum deflection \\
\hline$v$ & Poisson's ratio \\
\hline$v_{p}$ & Poisson's ratio for plastic strain increments \\
\hline$\rho$ & density of solid plate \\
\hline$\rho_{c}$ & effective density of sandwich core \\
\hline $\bar{\rho}_{c}=\rho_{c} / \rho$ & relative density of sandwich core \\
\hline$\sigma_{e}$ & effective stress \\
\hline$\sigma_{m}$ & mean stress \\
\hline$\sigma_{Y}$ & uniaxial tensile yield stress \\
\hline$\sigma_{Y}^{c}$ & compressive yield stress of sandwich core \\
\hline$\tau$ & duration of blast pressure \\
\hline$\tau_{Y}^{c}$ & shear yield stress of sandwich core \\
\hline
\end{tabular}

are compared with those of solid plates of the same material and total mass under identical impulse loads. Blast loads of sufficient magnitude are applied such that the plates undergo large permanent deflections. The material is taken to be elastic-perfectly plastic with no strain rate dependence. While detailed descriptions of the core will not be pursued in this first study, core properties representative of truss cores will be considered since they have the potential to achieve the combination of high strength and energy absorption necessary for effective performance of sandwich plates under blast loads.

The study is conducted within the framework of dynamic, finite strain plasticity for an elasticperfectly plastic solid with no rate dependence. Computations are preformed using the explicit time 
integration version of the commercially available code ABAQUS [2]. The simulations capture limits to deformation due to necking localization, however, no consideration will be made in this study of limits due to fracture. The focus will be on the comparison between sandwich plates and solid plates assuming each is able to withstand fracture. If the plates are constructed from a material with good ductility, this is tantamount to comparing them over the range of blast loads for which deformations are comparable.

To provide a basis of comparison, we start in Section 2 by investigating the elastic-plastic behavior of a clamped circular solid plate under blast loading, validating results in the literature $[3,4]$ and extending the results in several important respects. The class of loads is a uniform pressure applied for a finite period of time, including the limit for a zero time period corresponding to a uniform momentum impulse. The maximum deflection of the plate is determined as a function of the blast intensity. An important time scale emerges: the time required for the plate to attain the maximum deflection under the zero-period impulse. If the period of the blast pulse is longer that this characteristic time, the maximum deflection of the plate under the finite-period impulse may be significantly less than that for the corresponding zero-period impulse. Conversely, if the period of the blast pulse is sufficiently short compared to this characteristic time, the idealization of the blast as a zero-period impulse is justified.

The dynamic responses of clamped circular sandwich plates subject to uniformly distributed impulsive loads applied to one face sheet of the sandwich is studied in Section 3. For each impulse level, the maximum deflection is compared to that of a solid plate of the same mass. The core is envisioned to be a tetragonal truss structure and is modeled by a continuum plasticity relation developed previously for metal foam cores having a specific crushing strength. This crushing strength is matched to the corresponding strength of the truss core. The role of the strength of the core is studied via variations in its relative density, and some insight into an optimal design of the sandwich structure is obtained. A more extensive study of the blast performance of optimized sandwich plates subject to blast loads will be presented in subsequent papers.

The duration of an underwater or air blast pulse is typically on the order of $10^{-4} \mathrm{~s}(0.1 \mathrm{~ms})$. In all the examples of sandwich plates considered in this paper the characteristic response time referred to above is on the order of several ms. The performance assessment in this paper compares the responses of sandwich and solid plates of equal mass subject to identical zero-period impulses. It will be demonstrated in a sequel to this paper that this is a valid basis for comparison for air blasts. However, this approach underestimates the advantages of sandwich construction relative to solid plates for water blasts due to fluid-structure interaction.

\section{Dynamic response of a clamped circular solid plate}

As depicted in Fig. 1, a circular solid plate with outer radius $R$ and the uniform thickness $h$, which is fully clamped at the outer boundary, is subjected to a blast load.

\subsection{Idealized blast loading}

Explosives create a pressure wave with a triangular-like profile, known as a "blast" [5] as shown in Fig. 2(a). The blast exerts an impulse, $I$, on the circular plate, which is equal to the integral of 

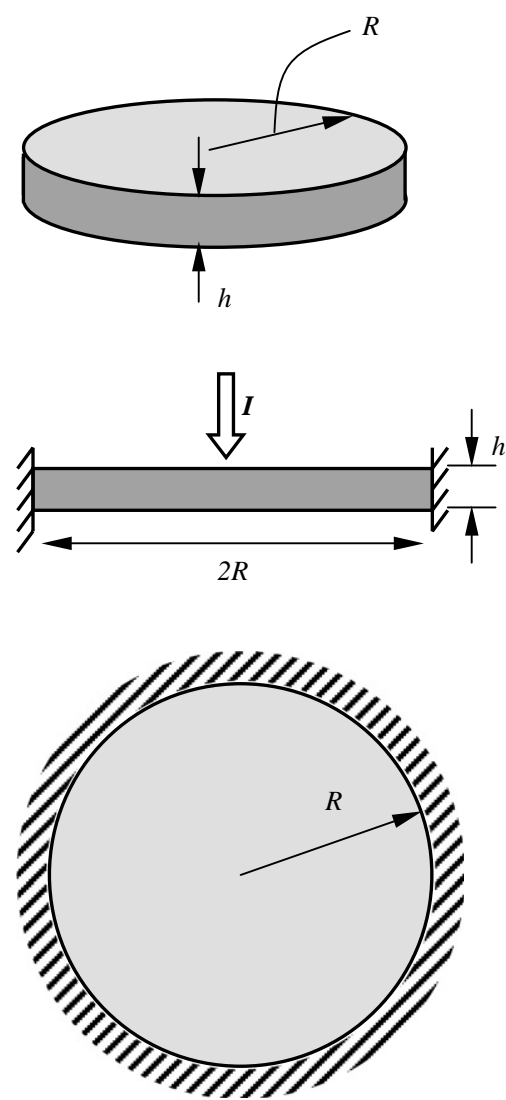

Fig. 1. A schematic diagram of the clamped circular solid plate under the blast loading.

the total force over time,

$$
I=\int p(r, \theta, t) \mathrm{d} A \mathrm{~d} t .
$$

The details of the spatial distribution and time history of the pressure depend on the position of the explosive center with respect to the plate. The pressure history will generally depend on the response of the plate itself, unless its duration is sufficiently short compared to response period of the plate, in which case it is as if the pulse hit a rigid wall. As mentioned above, the interaction between the pressure pulse and the plate response is not explicitly accounted for in this paper. The pressure pulse applied to the plate is idealized to be uniform over the entire plate with amplitude $p_{0}$ and duration $\tau$, as shown in Fig. 2(b):

$$
p= \begin{cases}p_{0}, & 0 \leqslant t \leqslant \tau, \\ 0, & t>\tau\end{cases}
$$

such that the impulse is $I=\pi R^{2} p_{0} \tau$. The impulse per area is $\hat{I}=I / \pi R^{2}=p_{0} \tau$. The limit of this family of loads corresponding to a zero-period impulse $(\tau \rightarrow 0)$ has initial momentum per area denoted 


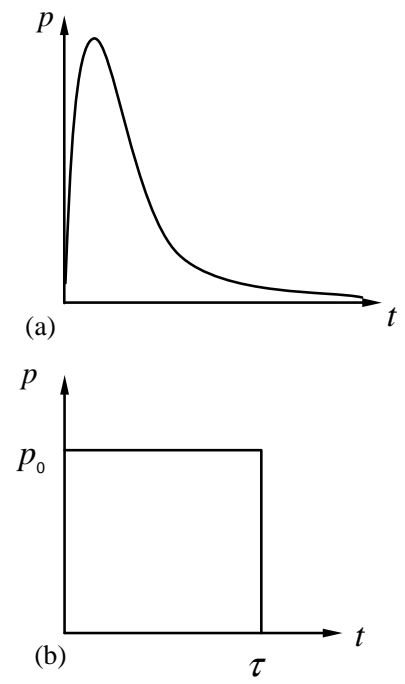

Fig. 2. (a) A schematic diagram of a pressure-time pulse; and (b) a rectangular pressure-time pulse.

by $\hat{I}$. For the solid plate of density $\rho$ and thickness $h$, the zero-period impulse $\hat{I}$ can be imposed as a uniform initial velocity $V_{0}$ prescribed throughout the plate where

$$
V_{0}=\frac{\hat{I}}{\rho h} .
$$

The dynamic behavior of the fully clamped circular plate loaded by the zero-period impulsive is investigated first, followed by the finite-period impulsive loading.

\subsection{Theoretical solutions for a clamped circular plate loaded impulsively}

Florence [6] has studied the behavior of a rigid-perfectly plastic circular plate which is fully clamped around an outer boundary and subjected to rectangular pressure pulse (2) applied uniformly over the plate, following earlier work of Wang and Hopkins [7] who examined the case of zero-period impulses (3). Both studies were restricted to linear bending kinetics, neglecting in-plane stretching. For the zero-period impulsive, Wang and Hopkins found the response duration, i.e. the time for the plate to reach its maximum deflection, to be

$$
T \cong 0.36 \rho V_{0} R^{2} / \sigma_{Y} h,
$$

where $\sigma_{Y}$ is the uniaxial tensile yield stress. The maximum permanent deflection at the plate center is

$$
\delta_{\max } \cong 0.28 \rho V_{0}^{2} R^{2} / \sigma_{Y} h .
$$

These theoretical solutions were developed for thin plates that undergo infinitesimal displacements and with plastic flow controlled by the Tresca yield criterion. 
Results such as those in (4) and (5) are limited to maximum deflections, $\delta_{\max }$, that are less than about $h / 2$, at which point in-plane stretching behavior begins to become important. Symonds and Wierzbicki [4] have examined the finite displacement response of impulsively loaded circular plates that are clamped around the entire boundary by neglecting bending and treating the plate as a membrane undergoing stretch. Their result for the maximum deflection can be written in the form

$$
\delta_{\max } \cong 0.83 V_{0} R \sqrt{\frac{\rho}{\sigma_{Y}}} .
$$

The results of the present simulations make contact with this result.

\subsection{Clamped circular plate loaded impulsively $(\tau=0)$}

In the present analysis, the elastic-plastic behavior of the plate is described by an elastic-perfectly plastic model with Young's modulus $E$, Poisson's ratio $v$ and uniaxial tensile yield stress $\sigma_{Y}$. The von Mises criterion is used to specify the yield surface. Because the deformations of the plate are dominated by inertia, the neglect of strain hardening does not lead to necking localization at small strain as would be the case if loads were applied quasi-statically. The simplicity of the material constitutive model is useful for the purposes of comparing behavior of solid and sandwich plates, although strain hardening and strain-rate sensitivity are clearly important in making final assessments.

A dimensional analysis implies that the response duration, $T$, can be expressed in functional form in terms of dimensionless combinations as

$$
T=R \sqrt{\frac{\rho}{\sigma_{Y}}} f\left(V_{0} \sqrt{\frac{\rho}{\sigma_{Y}}}, \frac{\sigma_{Y}}{E}, v, \frac{h}{R}\right),
$$

while the maximum deflection at the center of the plate can be written as

$$
\delta_{\max }=R F\left(V_{0} \sqrt{\frac{\rho}{\sigma_{Y}}}, \frac{\sigma_{Y}}{E}, v, \frac{h}{R}\right) .
$$

Finite element calculations have been performed simulating the dynamic response of the plate using the commercial code ABAQUS Explicit [2]. The plate is modeled as a three-dimensional, axisymmetric solid with a refined mesh near the outer boundary. For a set of given dimensionless parameters in (7) and (8), the response duration and maximum deflection have been computed. As mentioned above, $T$ is defined as the time for the plate to attain the maximum deflection. Motion of the plate continues for times greater than $T$, primarily as elastic vibrations. Results have been obtained over the following range of the parameters:

$$
0.04<V_{0} \sqrt{\frac{\rho}{\sigma_{Y}}}<0.6, \quad 0.0005<\frac{\sigma_{Y}}{E}<0.01, \quad v=0.3, \quad 0.005<\frac{h}{R}<0.06 .
$$

Over the above range of the parameters approximate formulas were developed to fit the computed values of $T / R \sqrt{\rho / \sigma_{Y}}$ and $\delta_{\max } / R$. These formulas are

$$
\frac{T}{R \sqrt{\rho / \sigma_{Y}}}=0.965\left[1-12\left(\frac{h}{R}\right)+95\left(\frac{h}{R}\right)^{2}\right]\left(1+0.4 \mathrm{e}^{-0.006 E / \sigma_{Y}}\right)
$$



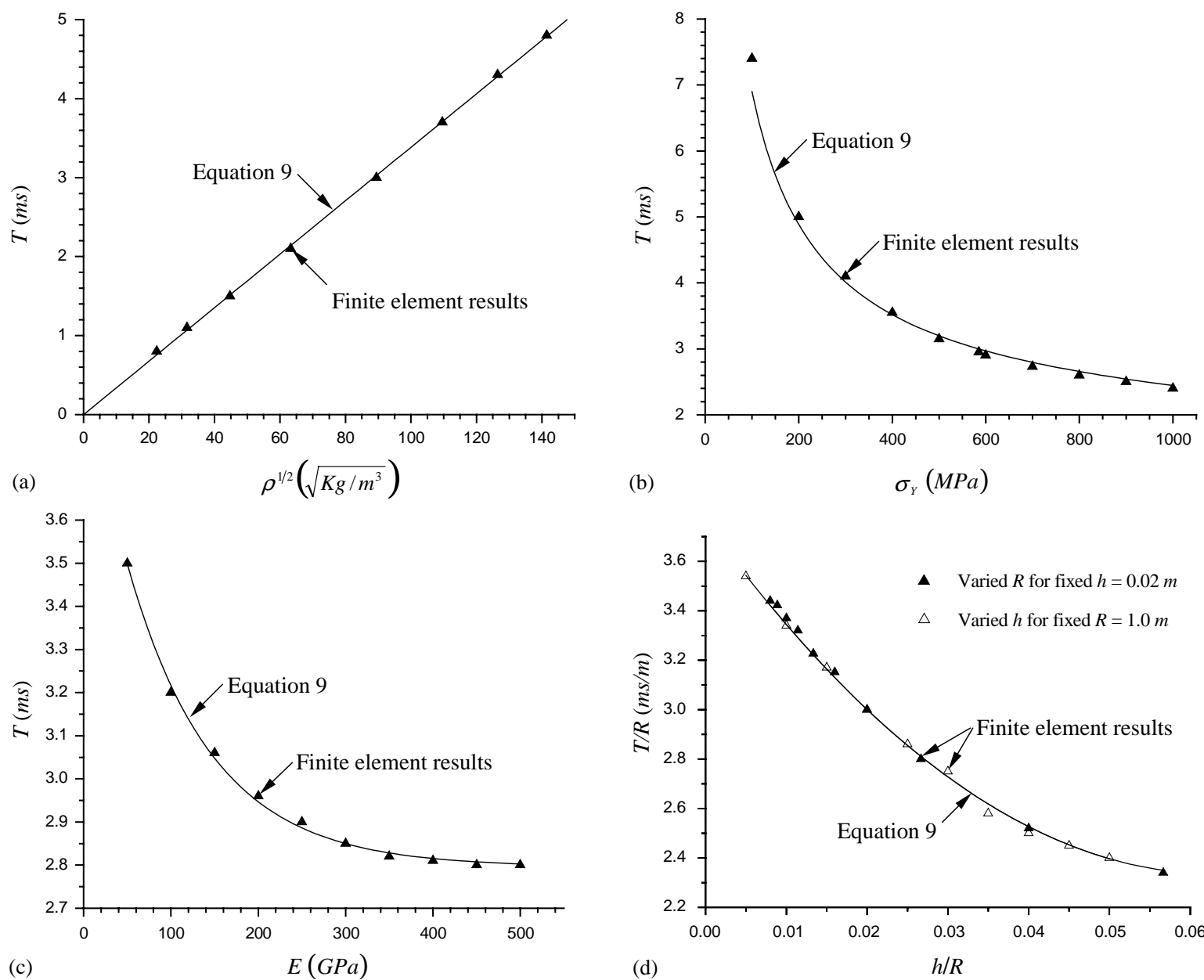

(b)

$$
\sigma_{Y}(M P a)
$$

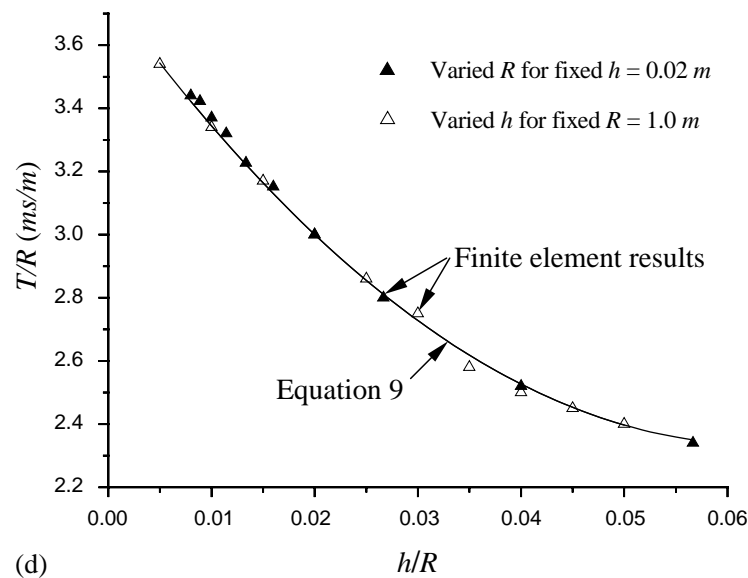

Fig. 3. A comparison of the approximate formula of response duration $T$ (solid curves from Eq. (9)) for various geometric and material parameters for the solid plate under zero-period impulse loading. The finite element results are plotted as the triangular points: (a) influence of density. Fixed parameters are: $E=200 \mathrm{GPa}, v=0.3, \sigma_{Y}=580 \mathrm{MPa}, V_{0}=31.25 \mathrm{~m} / \mathrm{s}$, $h=0.02 \mathrm{~m}$ and $R=1.0 \mathrm{~m}$; (b) influence of yield stress. Other fixed parameters are the same as those in Fig. $2 \mathrm{a}$ with $\rho=8000 \mathrm{~kg} / \mathrm{m}^{3}$; (c) influence of Young's modulus. Other fixed parameters are the same as those in Fig. 2a with $\rho=8000 \mathrm{~kg} / \mathrm{m}^{3}$; and (d) influence of $h / R$. Other fixed parameters are the same as those in Fig. $2 \mathrm{a}$ with $\rho=8000 \mathrm{~kg} / \mathrm{m}^{3}$. The solid triangular points present the finite-element results for different $R$ for the fixed thickness $h=0.02 \mathrm{~m}$; the hollow triangular points present those for different $h$ for the fixed radius $R=1.0 \mathrm{~m}$.

and

$$
\frac{\delta_{\max }}{R}=\left(V_{0} \sqrt{\frac{\rho}{\sigma_{Y}}}+0.84 \sqrt{\frac{\sigma_{Y}}{E}}-0.03\right)\left[1-8.3 \frac{h}{R}+25\left(\frac{h}{R}\right)^{2}\right] .
$$

The accuracy of the approximate formulas (10) and (11) is evident in the plots in Figs. 3a-d and $4 \mathrm{a}-\mathrm{d}$ where the results of the formulas are plotted as solid curves and results from specific finite 

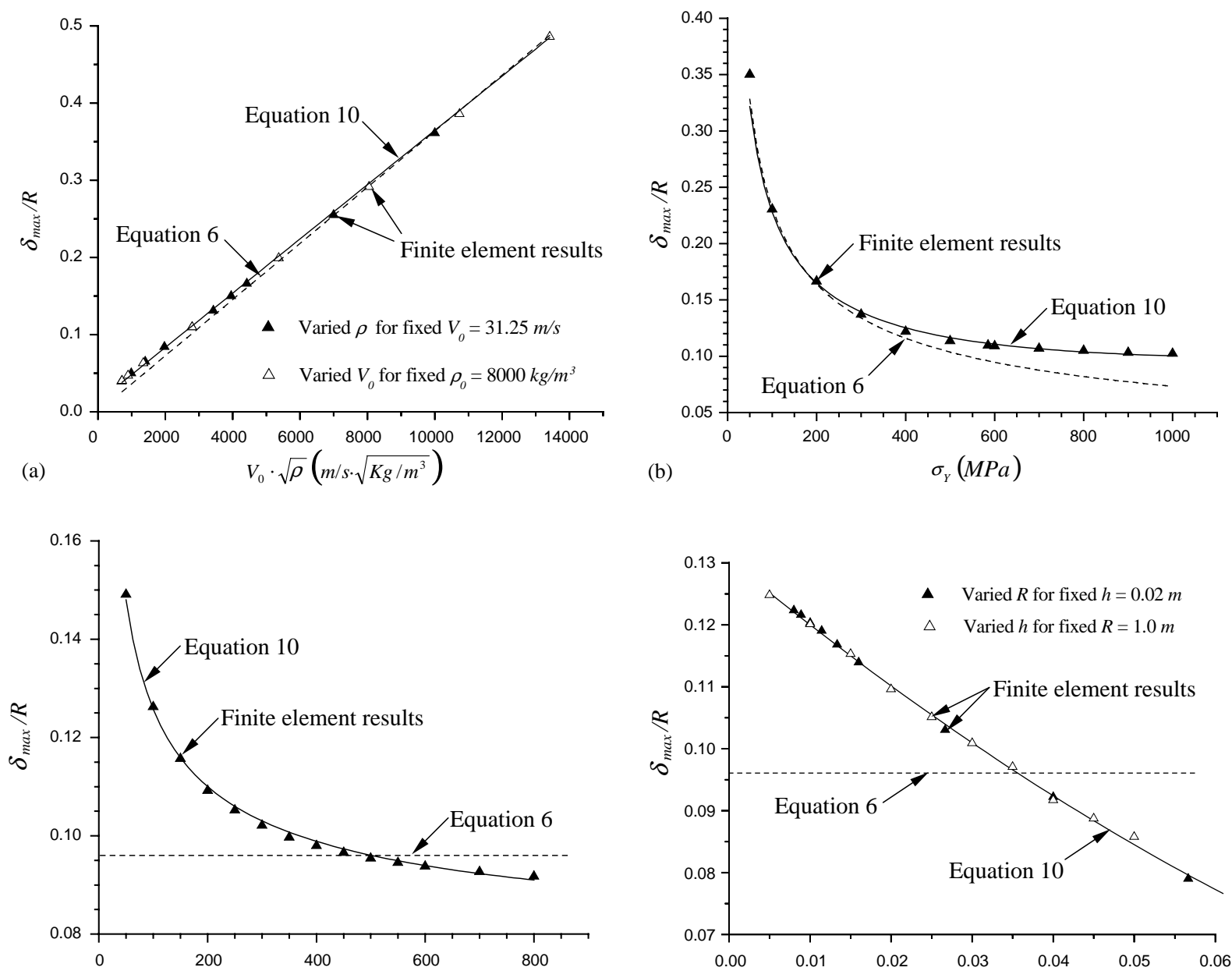

(c)

$E(G P a)$

(d)

Fig. 4. A comparison of the approximate formula of maximum deflection $\delta_{\max }$ (solid curves from Eq. (10)) for various geometric and material parameters for the solid plate under zero-period impulse loading. The finite-element results are plotted as the triangular points: (a) influence of initial velocity and density. Other fixed parameters are the same as those in Fig. 2a; (b) influence of the yield stress. Other fixed parameters are the same as those in Fig. $2 \mathrm{a} \mathrm{with} \rho=8000 \mathrm{~kg} / \mathrm{m}^{3}$; (c) influence of Young's modulus. Other fixed parameters are the same as those in Fig. $2 \mathrm{a}$ with $\rho=8000 \mathrm{~kg} / \mathrm{m}^{3} ; \mathrm{and}$ (d) influence of thickness and radius of the solid plate. Other fixed parameters are the same as those in Fig. 2a with $\rho=8000 \mathrm{~kg} / \mathrm{m}^{3}$. Solid triangular points present the finite-element results for different $R$ with $h=0.02 \mathrm{~m}$; the hollow triangular points present those for different $h$ with $R=1.0 \mathrm{~m}$.

element calculations are presented as triangular points. These comparisons span the range indicated in (9). Although not shown, $T /\left(R \sqrt{\rho / \sigma_{Y}}\right)$ is independent of $V_{0} \sqrt{\rho / \sigma_{Y}}$, to an excellent approximation over range (9). Additionally, Poisson's ratio, $v$, has almost no influence on $T /\left(R \sqrt{\rho / \sigma_{Y}}\right)$ and $\delta_{\max } / R$ over the range of the other parameters in (9). In four parts of Fig. 4, the prediction of solution (6) of Symonds and Wierzbicki has also been plotted as a dash line. It can 


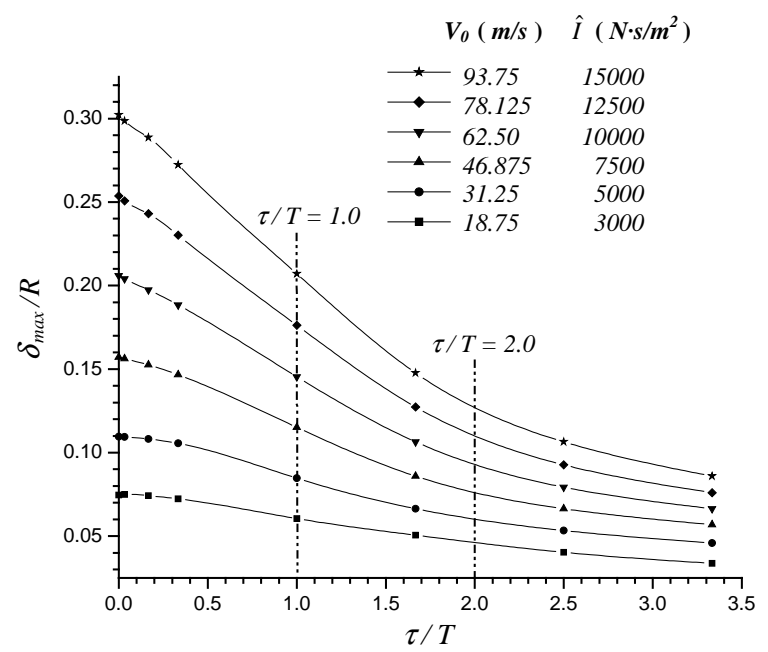

Fig. 5. Normalized maximum deflection versus the dimensionless impulse duration, $\tau / T$, for various impulse levels ( $V_{0}$ is the initial velocity for the zero-period impulse).

be seen that (6) accurately reflects the dependence on $V_{0} \sqrt{\rho}$ with $E$ and $\sigma_{Y}$ chosen as representative of a specific steel for $h / R=0.035$, but it does not capture the full dependence on $E$, $\sigma_{Y}$ and $h / R$.

\subsection{Clamped circular plate subjected to a finite period pressure pulse $(\tau>0)$}

A limited set of calculations has been carried out to reveal the relationship between the finite-period and zero-period impulse loads. The calculations have been carried out for a specific steel plate with the following properties: $R=1.0 \mathrm{~m}, h=0.02 \mathrm{~m}, \rho=8000 \mathrm{~kg} / \mathrm{m}^{3}, E=200 \mathrm{GPa}, v=0.3$ and $\sigma_{Y}=585 \mathrm{MPa}$. The finite element results for the finite period impulsive load are shown in Fig. 5. Each set of points connected by a solid curve corresponds to a prescribed level of impulse per unit area, $\hat{I}$. The initial velocity, $V_{0}$, associated with limiting zero-period impulse $(\tau=0)$ at the same $\hat{I}$ is shown in Fig. 5. The vertical axis represents the maximum deflection normalized by the radius of plate, while the horizontal axis is $\tau / T$. where $T$ is plate response duration for the zero-period impulse given by (10). For the plate in Fig. 5, $T=3 \mathrm{~ms}$.

For a given impulse level, $\hat{I}$, the maximum deflection occurs for the zero-period impulse. When $\tau / T=1$, the deflection is about two thirds that for the zero-period limit, while there is more than a $50 \%$ reduction of the deflection for $\tau / T>2$, although this does not account for any possible modification of the blast pulse due to the plate deflection. While no further examples have been studied, we expect the trends in Fig. 5 should be representative for most plates subject to blast loads. The results highlight the importance of the response duration, $T$, associated with the zero-period impulse. If the duration of the blast load is a small compared to $T$, as will be the case in most structural applications, response predictions based on the zero-period impulse should supply an accurate approximation, assuming fluid-structure interaction is taken into account. 


\section{Dynamic response of a clamped circular sandwich plate with a truss core}

In this section, the dynamic response of a clamped circular sandwich plate with a truss core will be studied for the case of a uniform distributed impulsive load applied to the (top) face sheet of the plate towards the blast. Attention will be restricted to zero-period impulses. The response of the sandwich plate will be compared with the response of the corresponding solid plate of Section 2 made from the same material and having the same radius and total mass. In this way, the competitive advantage or disadvantage of sandwich construction relative to the solid plate can be assessed. In this preliminary study, no effort has been made to identify optimal sandwich plates, although the role of the relative density of the truss core will be explored. The focus here is on truss cores because they have a relatively high specific crushing strength and energy absorbing capacity that is essential if the sandwich plate is to outperform solid construction. Other core construction, such as honeycomb, corrugated plate or woven wire, should also be considered as possible competitors. Indeed, perhaps the most important outcome of the present work, aside from demonstrating the relative advantage of sandwich construction, will be seen to be the need for further study to identify the best core geometry for blast resistant plates.

As before, the radius of the sandwich plate is $R$. The plate has two identical face sheets of thickness, $h_{f}$, bonded to a tetragonal truss structure core of thickness, $H_{c}$, as sketched in Fig. 6a. The face sheets and the truss elements are made from the same elastic-perfectly plastic material with density, $\rho$, Young's modulus, $E$, Poisson's ratio, $v$, and tensile yield stress, $\sigma_{Y}$. The yield surface of the face sheets is again taken to be the von Mises surface. The core is modeled using a continuum constitutive law for porous materials, as will be discussed below. For the sandwich plate, the uniform zero-period impulse, $I$, is applied as an initial uniform velocity, $V_{0}=I /\left(\rho \pi R^{2} h_{f}\right)$, of the top face sheet in Fig. 6a. Because the momentum impulse is applied to just one face sheet, the kinetic energy imparted to the sandwich plate is more than twice that imparted to the solid plate of

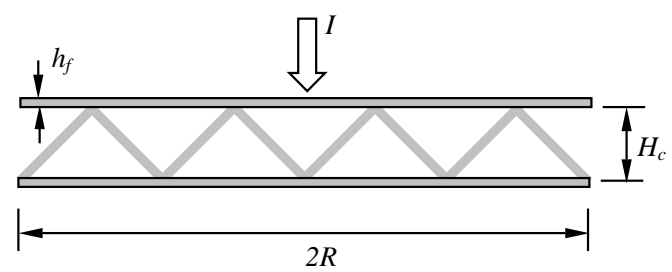

(a)

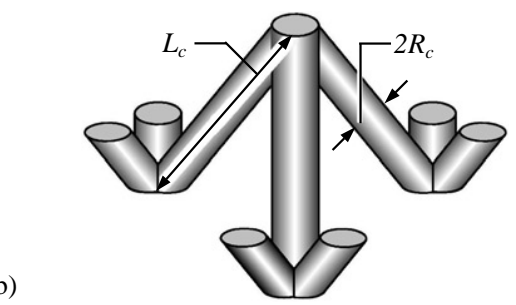

Fig. 6. (a) A schematic of a circular sandwich plate with a truss core subjected to impulsive loading; and (b) a tetragonal truss core unit. 
the same mass under the same impulse: i.e. $K E_{\text {sandwich }}=I^{2} /\left(2 \rho \pi R^{2} h_{f}\right)$ for the sandwich plate versus $K E_{\text {solid }}=I^{2} /\left(2 \rho \pi R^{2} h\right)$ for the solid plate. We return to this point later.

\subsection{Continuum constitutive model for sandwich core}

No attempt has been made to carry out calculations for the sandwich plate using full meshing of individual truss elements. Although such calculations might be feasible for a limited number of cases, they would be extremely large and would not permit an exploration of trends, which is the main goal here. Instead, a more practical approach is to model the core as a solid whose effective properties mimic those of the tetragonal truss between face sheets. At the present time, a constitutive model of this type for the tetragonal truss core has not been developed. Here, a constitutive model is adopted that was developed for metal foams by Deshpande and Fleck [8]. The model permits one to set the crushing strength of the core to match values appropriate to the tetragonal truss. Moreover, the model represents a material with high porosity similar to that of the truss core. The model is a dilatational plasticity relation that employs an isotropic yield surface specified by

$$
\hat{\sigma}=\sigma_{Y}^{c},
$$

where $\hat{\sigma}$ is an equivalent stress, defined by

$$
\hat{\sigma}^{2} \equiv \frac{1}{1+(\alpha / 3)^{2}}\left[\sigma_{e}^{2}+\alpha^{2} \sigma_{m}^{2}\right]
$$

Here, $\sigma_{e}=\sqrt{3 s_{i j} s_{i j} / 2}$ is the conventional effective stress with $s_{i j}$ as the stress deviator, and $\sigma_{m}=\sigma_{k k} / 3$ as the mean stress. The compressive yield stress, $\sigma_{Y}^{c}$, is a prescribed function of the equivalent plastic strain using data taken under uniaxial compression. Normality of plastic flow is assumed. For metal foams, the parameter $\alpha$ is usually chosen to produce a specific plastic Poisson's ratio $v_{p}=-\dot{\varepsilon}_{22}^{p} / \dot{\varepsilon}_{11}^{p}$ corresponding to uniaxial compression in the one-direction [8], i.e.,

$$
\alpha=3\left(\frac{1 / 2-v^{p}}{1+v^{p}}\right)^{1 / 2} \quad \text { or } \quad v_{p}=\frac{1 / 2-(\alpha / 3)^{2}}{1+(\alpha / 3)^{2}} .
$$

The flow stress, $\tau_{Y}^{c}$, in shear is related to that in compression at the same equivalent plastic by

$$
\tau_{Y}^{c}=\left[\frac{1+(\alpha / 3)^{2}}{3}\right]^{1 / 2} \sigma_{Y}^{c}
$$

\subsection{Properties of a tetragonal truss core and identification of $\sigma_{Y}^{c}$ and $\alpha$}

A tetragonal truss core element is configured as shown in Fig. 6b. The properties of all members of the core are identical with length $L_{c}$ and a solid circular cross-section of radius $R_{c}$. A regular tetragonal core with $H_{c} / L_{c}=\sqrt{2 / 3}$ has neighboring nodal points on the face sheets separated by $L_{c}$. The relative density of this core, $\hat{\rho}_{c}$, defined as the ratio of equivalent core density, $\rho_{c}$, to the density of the material, $\rho$, can be expressed in terms of $L_{c}$ and $R_{c}$ as

$$
\bar{\rho}_{c}=\frac{\rho_{c}}{\rho}=3 \sqrt{2 \pi}\left(\frac{R_{c}}{L_{c}}\right)^{2} .
$$


The crushing yield stress of regular tetragonal core, $\sigma_{Y}^{c}$, is defined as the normal force per unit area applied to the face sheet required bringing the truss members to compressive yield. It is related to the relative density by Deshpande et al. [9]

$$
\sigma_{Y}^{c}=\frac{2}{3} \bar{\rho}_{c} \sigma_{Y}
$$

Similarly, the Young's modulus of the core under normal loading of the face sheets, $E_{c}$, is

$$
E_{c}=\frac{4}{9} \bar{\rho}_{c} E \text {. }
$$

Tests on normal compression of tetragonal truss core elements [10] have shown that (17) provides a reasonably accurate estimate of the maximum crushing strength of the core, underestimating this strength when strain hardening is appreciable. Following attainment of the maximum, the crushing stress gradually falls but still retains a large fraction of the maximum strength at overall compressive strains above $10 \%$. In the present simulations, $\sigma_{Y}^{c}$ in the core constitutive model is taken as (17), independent of the effective plastic strain for strains less than $60 \%$. For effective plastic strains above $60 \%$, a linear hardening behavior with a large hardening rate is adopted. In the constitutive model, we have taken $\alpha=3 / \sqrt{2}$ which, by (15), gives $v_{p}=0$. This choice corresponds to metal foams with a relative density typically in the range of $5-10 \%$ [11]. The choice $v_{p}=0$ for modeling the tetragonal core is motivated by the fact that its compressive behavior normal to the faces is essentially independent of deformations parallel to the faces. By (15), the constitutive model implies the core shear strength is $\tau_{Y}^{c}=\sigma_{Y}^{c} / \sqrt{2}$. The constitutive model overestimates the shear strength corresponding a direct calculation of yield of the regular tetragonal core when the face sheets are sheared with no normal stress [9]: $\tau_{Y}^{c}=\bar{\rho}_{c} \sigma_{Y} / 3 \sqrt{2}$ or $\tau_{Y}^{c}=\sigma_{Y}^{c} / 2 \sqrt{2}=0.353 \sigma_{Y}^{c}$ (see also shear tests of core elements which validate this result [10]).

Subsequent work with more realistic constitutive representations for the core will be required to verify the findings presented here which are based on a simplified representation of crushing behavior and overly high strengths for the core in shear and in-plane stretching. It is expected that the present results will not be changed significantly by lower core shear strength since the face sheets are held rigidly at their perimeter, tending to suppress overall shearing motion between the faces. The characteristic of the core of primary importance in the present examples is its crushing strength, and it will be shown that most of the energy absorbed in the core is due to crushing not stretching. Nevertheless, models allowing for better representations of core behavior are needed, as are models that account for the effects of local inertia, strain hardening and strain rate-sensitivity in the core. The influence of strain hardening and strain rate-sensitivity in the face sheets should also be studied.

The total mass of the sandwich plate is $M_{\text {sandwich }}=\rho \pi R^{2}\left(2 h_{f}+\bar{\rho}_{c} H_{c}\right)$, while the mass of a solid plate of thickness $h$ is $M_{\text {solid }}=\rho \pi R^{2} h$. The response of the sandwich plate will be compared to the response of the solid plate of the same radius and mass subject to the same loading. Thus, $M_{\text {sandwich }}=M_{\text {solid }}$ requires

$$
2 h_{f}+\bar{\rho}_{c} H_{c}=h .
$$

In the calculations carried out below both the face sheets and the truss members are made from a moderately high strength steel with $\rho=8000 \mathrm{~kg} / \mathrm{m}^{3} \sigma_{Y}=500 \mathrm{MPa}, E=200 \mathrm{GPa}$ and $v=0.3$. The relative density of the regular tetragonal core is given by (16). The compressive yield stress 


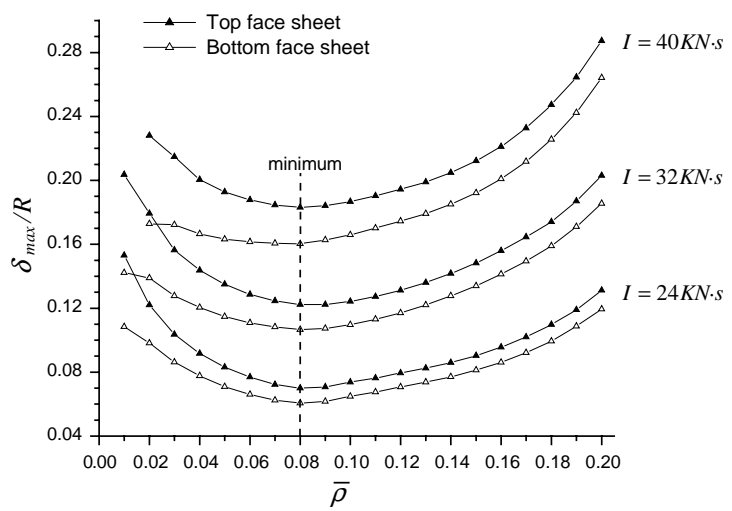

Fig. 7. The normalized maximum deflection of the top and bottom face sheets of a sandwich plate versus the relative density of core for three zero-period impulses. All plates are of equal mass, as detailed in the text.

of the continuum constitutive model is given by (17), and $\alpha=3 / \sqrt{2}$ in (13). The elastic properties of the core are modeled as isotropic with Young's modulus (18) and Poisson's ratio, $v_{c}=0$. The example analyzed in this section has $R=1.0 \mathrm{~m}$ and $M_{\text {sandwich }}=M_{\text {solid }}=628.32 \mathrm{~kg}$ corresponding to $h=2.5 \mathrm{~cm}$ for the solid plate. The thickness of the core of the sandwich plate is fixed at $H_{c}=10 \mathrm{~cm}$, but the relative core density, $\bar{\rho}_{c}$, is varied over the range from 0.01 to 0.2 . As $\bar{\rho}_{c}$ varies, so does $h_{f}$ according to (19). For this choice of parameters, the relative density must satisfy $\bar{\rho}_{c}<0.25$ if $h_{f}>0$.

Four-node axisymmetric elements with reduced integration are used in the calculations. A uniform mesh is generated such that there are 5 and 30 divisions through the thickness of the face sheets and the core, respectively, but 80 divisions along the radius for the whole plate. Additional studies showed that refined meshes with more elements did not appreciably improve the accuracy of numerical results. For the specific model, the numerical error for the maximum deflection based on the present meshing is believed to be less than $1 \%$.

\subsection{Numerical results for sandwich plates and comparison with solid plates of equal mass}

Fig. 7 presents the normalized maximum deflection $\delta_{\max } / R$ attained at the center of each face sheet as a function of the relative core density, $\bar{\rho}_{c}$, for three levels of zero-period impulses applied uniformly to the top face sheet. Each triangular point represents a calculation for a specific value of $\bar{\rho}_{c}$. All of the sandwich plates in Fig. 7 have the same mass. The top face sheet deflects more than the bottom sheet due to compaction of the core. Typically, the nominal compressive strain in the core, defined as compressive deformation per unit core thickness, is between $10 \%$ and $20 \%$, except for the lowest core densities where the compaction strain reaches as much as $50 \%$. At each of the three blast impulse levels, the smallest maximum deflection occurs for a relative core density $\bar{\rho}_{c} \approx 0.08$. At lower core densities and, therefore, higher face sheet mass, the core undergoes so much compaction that it is not able to maintain the spacing of the face sheets such that the sandwich effect is eroded. At core densities above $\bar{\rho}_{c}=0.08$, core mass is added at the expense of face sheet mass, rendering the faces too weak to be effective. A full optimization, wherein the core thickness, 


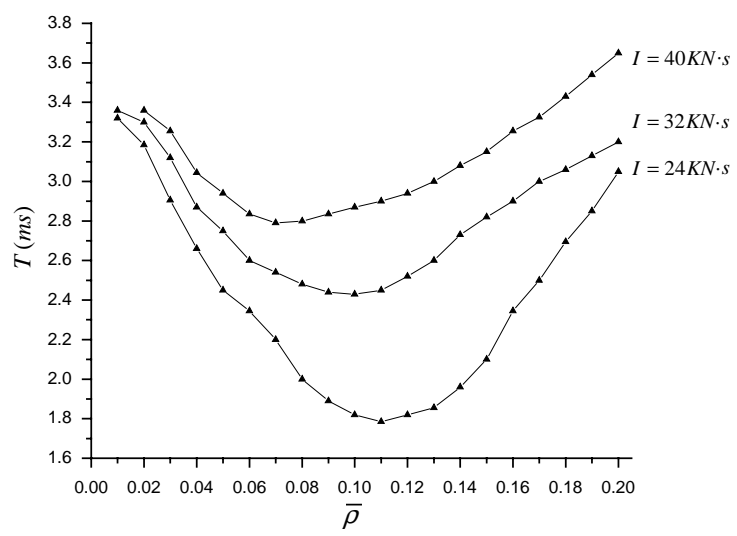

Fig. 8. The response duration of the sandwich plates in Fig. 7 .

$H_{c}$, is also varied, has not been carried out, and, thus, no conclusion should be drawn as to the optimal core density.

The plates in Fig. 7 are not likely to sustain impulses greater than $I=40 \mathrm{kN} \mathrm{s}$ because of unacceptably large deflections, even if necking or fracture did not intervene. Companion plots of the time, $T$, to attain the maximum deflection are given in Fig. 8. The corresponding time for the solid plate, $T=3 \mathrm{~ms}$, essentially independent of $I$. The shortest response time for the sandwich plate is less than that for the solid plate and is associated with a relative core density close to that giving the smallest deflection. Note that even the shortest response time is still long compared to the typical blast pressure duration of a fraction of $10^{-4} \mathrm{~s}$.

To reveal the relative advantage of sandwich plate construction, the results in Fig. 7 have been replotted in Figs. 9a and b by normalizing the maximum deflection of the sandwich plate by the maximum deflection of the solid plate of the same mass, $\left(\delta_{\max }\right)_{\text {solid }}$, that is subject to the same zero-period impulse. The greatest relative performance advantage of the sandwich plate is seen for the lower impulse levels, although there is still an advantage at the highest level of impulse considered. Over the range of impulses considered, the maximum deflection of a sandwich plate with $\bar{\rho}_{c} \approx 0.08$ is between $50 \%$ and $85 \%$ of the corresponding solid plate. As stated earlier, at times subsequent to attaining the maximum deflection, the plate continues to vibrate elastically, but most of the energy imparted by the impulse is dissipated in plastic deformation by the time when the maximum deflection is attained (typically more than $85 \%$ ).

Apart from the small amount of energy stored as residual stress or as vibratory motion, essentially all the initial kinetic energy imparted to the plates is absorbed by plastic deformation. In comparing the performance of the sandwich plate with the solid plate it is important to distinguish between the initial impulse imparted to the plates and their initial kinetic energies. In this study, we have deliberately compared the plate responses under identical zero-period momentum impulses, consistent with the blast pressure pulse acting for a time short compared to the response time of the plates. As noted earlier, the initial kinetic energies imparted to the two plates are different because the momentum pulse is distributed uniformly throughout the entire solid plate at $t=0$, while the momentum impulse is initially confined to the top face sheet in the case of the sandwich plate. By (19), the ratio of the 

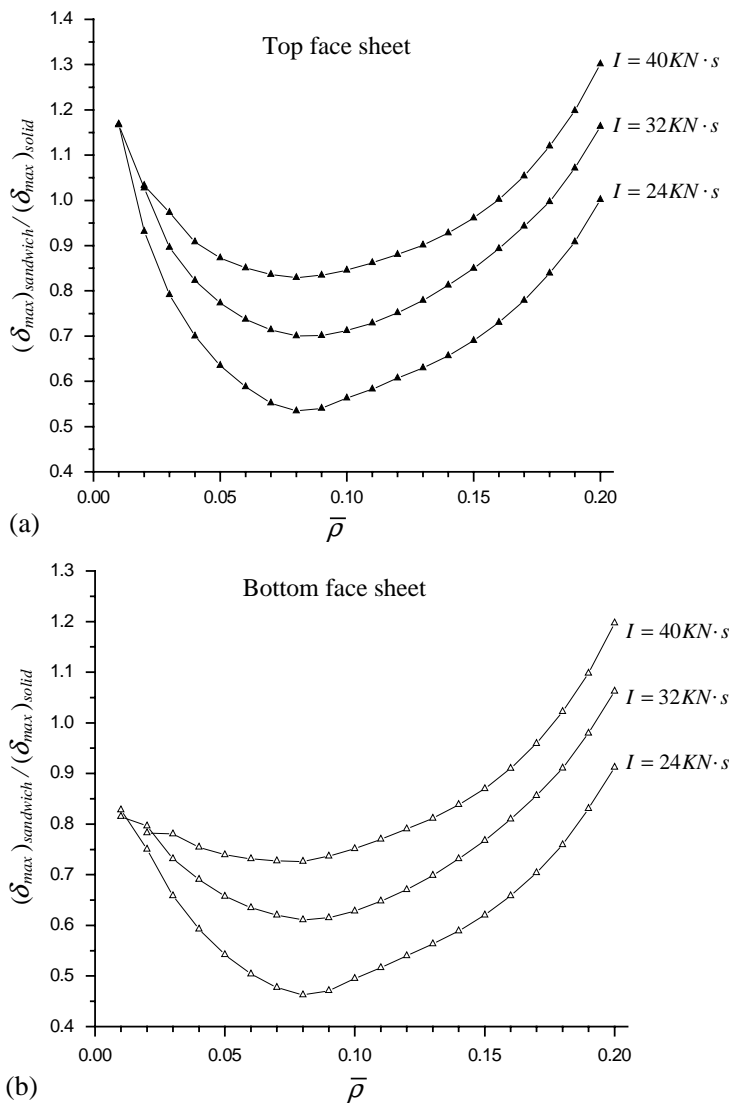

Fig. 9. The ratio of the maximum deflection of a sandwich plate to that of a solid plate of identical mass for three zero-period impulses: (a) top face sheet; and (b) bottom face sheet.

initial kinetic energies is

$$
\frac{K E_{\text {solid }}}{K E_{\text {sandwich }}}=\frac{1}{2}\left(1-\bar{\rho}_{c} \frac{H_{c}}{h}\right) .
$$

For the example in Fig. 9, with $\bar{\rho}_{c}=0.08, K E_{\text {solid }} / K E_{\text {sandwich }}=0.34$. Therefore, it follows from Fig. 9 that the sandwich plates absorb about three times as much energy as their solid counterparts, while at the same time experiencing smaller deflections.

\subsection{Energy absorption in sandwich plates}

Significantly larger energy absorption in the sandwich plate relative to the corresponding solid plate can be attributed to several factors that are revealed by the time histories of plastic dissipation in the face sheets and core of the sandwich plate in Fig. 10. The time histories are for the plate with $\bar{\rho}_{c}=0.08$ and $I=32 \mathrm{kN} \mathrm{s}$. In the first phase of the response, lasting until $t \approx 0.2 \mathrm{~ms}$, the top face sheet flies into the core, resulting in core compaction and significant energy dissipation. By the end of the first phase, the two face sheets are moving at approximately the same velocity and most 


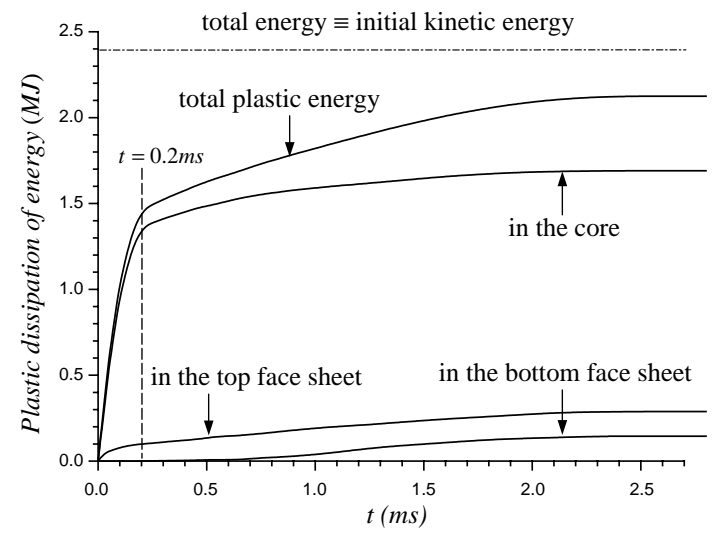

Fig. 10. The time histories of plastic dissipation in the face sheets and core of the sandwich plate in Fig. 7 with $\bar{\rho}_{c}=0.08$ and $I=32 \mathrm{kN} \mathrm{s}$.

core compaction ceases. As the plate deflection increases, plastic dissipation is increasingly due to stretch. Further details are discussed below.

(1) Core compression constitutes a major contribution to energy dissipation. Although the crushing strength of the core is only a small fraction of $\sigma_{Y}$, the average compaction strain is between $10 \%$ and $20 \%$. In the stretching phase of the response, Fig. 10 indicates there is additional plastic dissipation of energy in the core due to in-plane stretch, although this is only a small fraction of the dissipation during crushing. Stretch dissipation in the core is an artifact of the constitutive model used to represent the core in the present study. It would not occur if the tetragonal core were more accurately modeled.

(2) After the compaction phase has ended and as long as deflections, $\delta$, are still less than about the sandwich thickness, $H_{c}$, the response of the sandwich plate is dominated by bending. The top face sheet experiences in-plane compression while the bottom face undergoes in-plane tension. As even larger deflections develop, stretching takes over and the in-plane stresses become tensile in both faces. Thus, the top face sheet undergoes reversed plastic deformation with compressive straining followed by tensile straining resulting in enhanced energy absorption. The solid plate undergoes a similar transition from bending to stretching but at much smaller deflections that are on the order of $h$. No plastic deformation on the top surface of the solid plate occurs during the bending phase of the corresponding solid plates. Moreover, in the bending regime, the bending strength of the sandwich plate far exceeds that of the solid plate.

\subsection{Strains in the face sheets and core}

Apart from large strains occurring in the top face sheet at the outer edge of the plate, the strains in the face sheets are for the plates in Fig. 7 are modest. The maximum stretching strain in the bottom face sheet at attainment of $\delta_{\max }$ for $\bar{\rho}_{c} \approx 0.08$ is $0.62 \%$ for $I=24 \mathrm{kN} \mathrm{s}, 2.9 \%$ for $I=32 \mathrm{kN} \mathrm{s}$, and $5.1 \%$ for $I=40 \mathrm{kN}$ s. Figs. $11 \mathrm{a}$ and $\mathrm{b}$ display radial distributions of the radial strain, $\varepsilon_{r r}$, and transverse shear strain, $\varepsilon_{r z}$, of the top and bottom face sheets corresponding to the attainment of 


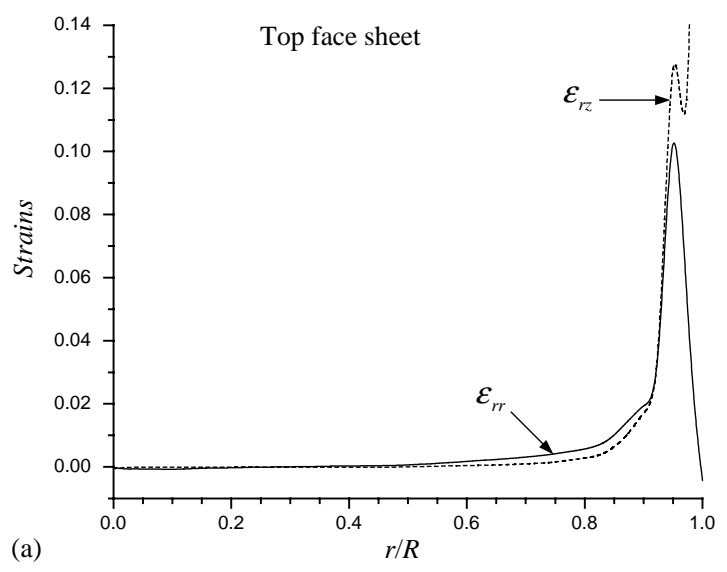

(a)

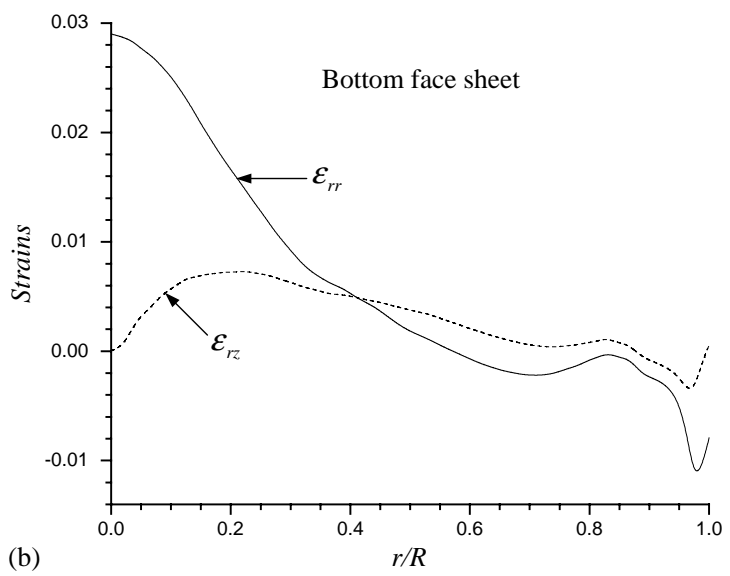

Fig. 11. The distributions of the radial strain $\varepsilon_{r r}$ and transverse shear strain $\varepsilon_{r z}$ in the top face sheet (a) and bottom face sheet (b).

$\delta_{\max }$ for the sandwich plate with $\bar{\rho}_{c}=0.08$ and $I=32 \mathrm{kN} \mathrm{s}$. The strain distributions in the bottom face sheet are unexceptional with no component exceeding 0.03. Strain localization involving both stretching and transverse shear strain components occurs at the outer edge of the top plate. The large strains might well result in fracture of the top face sheet at the outer edge. Due to necking down of the top face sheet at this location, the load carrying capacity of this sheet is already reduced. Nevertheless, the bottom face sheet has not experienced appreciable increases in strain at the outer edge and is fully intact.

As remarked in Section 1, the numerical code used in the present study is capable of computing necking localization, as illustrated in Fig. 11. In spite of the fact that an elastic-perfectly plastic representation for the material has been used, no other tendency for necking has been observed. This is thought to be due to the fact that the deformations are dominated by inertia. Quasi-static simulations of uniform pressure loading of the same elastic-perfectly plastic plates indicate a much higher susceptibility to necking localization. The localization of strain at the outer edge of the top face sheet is clearly an issue of concern to be addressed in subsequent work. Factors not taken into 
account here such as strain hardening and material rate-sensitivity will influence the tendency to localization, as will details of the core constitutive behavior. More realistic support conditions will also be a factor in localization.

Crushing localization in the core also occurs. For the largest blast, $I=40 \mathrm{kN} \mathrm{s}$, crushing strains on the order of $50 \%$ occur across the entire radial extent of the plate in a zone of thickness roughly $0.2 H_{c}$ just below the top face sheet. About 12 elements span this localization region in the thickness direction. Outside this zone of crushing localization the strain is much less (but still well into the plastic range) such that the average compaction strain in the core is $15 \%$. This localization does not have a direct interpretation for a truss core. It is, instead, an artifact of replacing the discrete core truss elements by a continuum model. Deformations akin to a crushing localization may indeed occur in a truss core, but the present model cannot be expected to capture them. The present model correctly accounts for energy dissipation in the core under the assumption that the crushing strength is constant at $\sigma_{Y}^{c}$, even when localization occurs. We have explored the robustness of the comparison between sandwich and solid plate in Fig. 9 by repeating selected calculations with the introduction of a modest amount of strain hardening into the crushing stress-strain curve for the core. The strain hardening reduces, but does not eliminate, the localization. More significantly for present purposes, the results presented in Fig. 9 are virtually unaffected by these alterations.

\section{Concluding remarks and recommendations for further research}

The preliminary study conducted in this paper suggests that sandwich plates with sufficiently strong cores have the potential to sustain substantially larger uniform impulses than solid plates of the same material and weight. A limited number of systematic comparisons for clamped circular plates have been made to highlight the relative advantages of sandwich construction, but no effort has been made to optimize the sandwich plates. Nor has there been any effort to explore any core geometry other than the tetragonal truss core. Strain hardening and strain-rate sensitivity are almost certainly of importance but have not been taken into account in either the face sheets or the core.

The present study has been hindered by the lack of an accurate continuum constitutive relation that can be used to model the core. Strength characteristics of the tetragonal core are highly anisotropic. The prediction of a crushing localization highlights the need for development of constitutive relation for the core that is able to more faithfully represent, or mimic, details of the deformation of the truss elements. The core model used here ignores several factors that are likely to be important and, possibly, beneficial to the capabilities of the sandwich plate. These include strain hardening, strain rate-sensitivity and, especially, local "inertial stiffening" of individual truss elements. The development of a relation that captures anisotropy, rate-dependence and inertial effects in the core is a priority.

Optimization studies at several levels are essential if the most effective sandwich structures are to be discovered. The present study has bought out the combined importance of core strength and energy absorption, highlighting the need for further research to identify the most effective core geometry (truss, honeycomb, etc.). In addition to core geometry, core thickness and relative density should be considered in the optimization process. There is a complicated interplay between face sheet thickness, initial kinetic energy, energy absorption and fluid-structure interaction. As emphasized in the paper, a well-designed sandwich plate out performs a solid plate of the same weight even though 
the sandwich plate must absorb more than twice the energy. In the paper to follow this one, it will be shown that there is a significant additional relative advantage of sandwich construction when blasts occur in water due to fluid-structure interaction. The possibility of designs having face sheets of different thickness should be considered. Among sandwich plates of constant weight subject to a specified zero-period impulse, the thinner the face sheet towards the blast, the higher the initial kinetic energy imparted to structure. This energy must be absorbed by plastic deformation of the entire plate. By increasing the thickness of the face sheet towards the blast relative to the other sheet, one may be able to achieve a more effective design for blasts in air. The situation for water blasts is likely to be different. Optimization clearly has a role to play here as well.

Finally, it must be emphasized that the most effective designs are likely to depend in a significant way on additional aspects not considered here. These include other plate shapes, support conditions, and other loading conditions such as localized blasts.

\section{Acknowledgements}

This work has been supported in part by the ONR under grants GG10376-114934 and N00014-021-0700 and in part by the Division of Engineering and Applied Sciences, Harvard University.

\section{References}

[1] Mäkinen K. Underwater shock loaded sandwich structures. Doctoral thesis, Department of Aeronautics, Royal Institute of Technology, Sweden, 1999.

[2] ABAQUS/Explicit User's Manual. Version 5.8, Hibbit, Karlsson and Sorensen Inc., 1998.

[3] Jones N. Structural impact. Cambridge: Cambridge University Press, 1989.

[4] Symonds PS, Wierzbicki T. Membrane mode solutions for impulsively loaded circular plates. Journal of Applied Mechanics 1979;46(1):58-64.

[5] Smith PD, Hetherington JG. Blast and ballistic loading of structures. Oxford: Butterworth-Heinemann, 1994.

[6] Florence AL. Clamped circular rigid-plastic plates under blast loading. Journal of Applied Mechanics 1966;33(2): 256-60.

[7] Wang AJ, Hopkins HG. On the plastic deformation of built-in circular plates under impulsive load. Journal of the Mechanics and Physics of Solids 1954;3:22-37.

[8] Deshpande VS, Fleck NA. Isotropic constitutive models for metallic foams. Journal of the Mechanics and Physics of Solids 2000;48:1253-83.

[9] Deshpande VS, Fleck NA, Ashby MF. Effective properties of the octet-truss lattice material. Journal of the Mechanics and Physics of Solids 2001;49:1747-69.

[10] Chiras S, Mumm DR, Evans AG, Wicks N, Hutchinson JW, Dharmasena K, Wadley HNG, Fichter S. The structural performance of near-optimized truss-core panels. International Journal of Solids and Structures 2002;39:4093-115.

[11] Ashby MF, Evans AG, Fleck NA, Gibson LJ, Hutchinson JW, Wadley HGN. Metal foams: a design guide. Boston: Butterworth-Heinemann, 2000. 